\title{
A complexidade em alergologia pediátrica: o doente polissensibilizado
}

\section{The complexity in pediatric allergology: the polysensitized patient}

\author{
João Virtuoso $\left(\mathbb{D}{ }^{1} \bowtie\right.$, Íris Santos Silva $(\mathbb{D})^{1}$, Pedro Guerra $(\mathbb{D})^{1}$, Rita S. Oliveira $(\mathbb{D})^{1}$
}

${ }^{1}$ Hospital Sousa Martins, Unidade Local de Saúde da Guarda, Serviço de Pediatria. Guarda, Portugal.

Como citar este artigo (How to cite this article):

Virtuoso J, Silva IS, Guerra P, Oliveira RS. A complexidade em alergologia pediátrica: o doente polissensibilizado (The complexity in pediatric allergology: the polysensitized patient). Sci Med. 2019,29(4):e33629. https://doi.org/10.15448/1980-6108.2019.4.33629

\section{RESUMO}

OBJETIVOS: No presente estudo os autores pretendem demonstrar as vantagens do diagnóstico molecular em imunoalergologia atráves da descrição de um caso clínico.

DESCRIÇÃO DO CASO: Apresentamos o caso de uma criança, do sexo masculino, 9 anos de idade seguido em consulta de imunoalergologia pediátrica com os diagnósticos de asma persistente moderada, rinite persistente, eczema atópico e alergia alimentar com manifestações graves (vários episódios de anafilaxia), cujo estudo do perfil molecular de sensibilização alérgica contribuiu para melhor conhecimento do doente, adequação terapêutica e, inclusive, prognóstica.

CONCLUSÕES: O estudo molecular alergológico, conforme análise do caso em estudo, permite o estabelecimento e escalonamento em termos de gravidade de um perfil de sensibilização mais individualista, que é sobretudo importante no doente polissensibilizado e com relato de alergias respiratórias e alimentares.

DESCRITORES: Diagnóstico, alergologia, pediatria.

\section{ABSTRACT}

AIMS: In the present study, the authors intend to demonstrate the advantages of molecular diagnosis in immunoallergology through the description of a clinical case.

CASE DESCRIPTION: We present the case of a 9-year-old male, followed by pediatric immunoallergology with the diagnoses of moderate persistent asthma, persistent rhinitis, atopic eczema and food allergy with severe manifestations (several episodes of anaphylaxis). molecular profile of allergic sensitization contributed to better knowledge of the patient, therapeutic and even prognostic adequacy.

CONCLUSIONS: The molecular allergy diagnostic tests, according to the analysis of the present case, allows the establishment and escalation in terms of severity of a more individualistic sensitization profile, which is especially important in the patient who is polysensitized and with reports of respiratory and food allergies.

KEYWORDS: Diagnostic, allergology, pediatrics. 
Abreviaturas: Ig E, imunoglobulina E; ISU, Isac unidades estandardizadas.

\section{INTRODUÇÃO}

Uma história clínica detalhada, conjuntamente com a realização de teste cutâneos por picada e o doseamento de imunoglobulinas E (IgE) alergénio-especificas, constituem a base diagnóstica de primeira linha em alergologia. No entanto, durante as últimas décadas, os desenvolvimentos tecnológicos permitiram uma melhoria significativa das ferramentas de diagnóstico laboratorial, sobretudo com o acesso ao estudo da caracterização dos componentes moleculares alergênicos [1], cuja utilização se tem vindo a generalizar.

Os alergênios moleculares condicionam uma definição mais precisa do perfil individual de sensibilização, permitindo concluir se estamos perante um caso de sensibilização a um alérgeno genuíno ou, por outro lado, se se trata de reação cruzada; permite ainda identificar moléculas consideradas de risco, aquelas cuja presença está associada com reacções de maior potencial de gravidade clínica. Extrapolando estes dados para a prática clínica, o uso do estudo alergológico molecular está implicado na melhor especificação de seleção dos componentes da imunoterapia específica, quando é lícito o seu uso e, o seu potencial diagnóstico é substancialmente útil em estudos de alergia alimentar, no sentido de identificar com maior precisão quais os componentes da dieta que devem ser evitados, assim como eventuais reações cruzadas existentes (como, por exemplo, no caso particular dos síndromes pólenalimento ou látex-alimento) $[2,3]$.

O diagnóstico molecular de alergia pode ser realizado pela determinação do nível de anticorpos $\mathrm{IgE}$ a alérgenos individuais, tecnologia denominada de singleplex, ou através de uma plataforma desenvolvida para medição simultânea num único ensaio de $\operatorname{IgE}$ específicas para um painel de alérgenos purificados, naturais ou recombinantes, de variadas fontes alergénicas, tecnologia designada de multiplex $[4,5]$.

Dentre este último grupo inclui-se o Immuno $\mathrm{CAP}^{\circledR}$ Isac (Immuno Solid Phase Allergen Chip), um teste in vitro que visa identificar e determinar semiquantitativamente a presença de $\operatorname{IgE}$ no soro ou no plasma humano para 112 diferentes componentes proveniente de 51 fontes alergênicas. O exame consiste em um imunoensaio, onde os componentes alergênicos ficam imobilizados num substrato sólido, na forma de microarray, e são incubados com $20 \mu \mathrm{L}$ de soro do paciente; a ligação entre esses anticorpos e os componentes alergénicos é identificada pela adição de um anticorpo anti-IgE, com marcação fluorescente. Com a existência de um software adequado, a intensidade de fluorescência é mensurável, sendo os resultados expressos em Isac Standardized Units (ISU), com cut-off de positividade de 0,3 ISU $[2,3,4]$.

No presente artigo, os autores descrevem um caso clínico de um doente pediátrico cuja investigação do perfil alergológico molecular contribuiu para uma melhoria de caracterização diagnóstica e adequação terapêutica.

\section{RELATO DE CASO}

Menino de 9 anos de idade, raça caucasiana, realiza consulta com alergologia pediátrica com os diagnósticos de alergia alimentar, asma persistente ligeira, rinite persistente moderada e dermatite atópica. Apresenta antecedentes pessoais de eczema atópico desde o primeiro ano de vida e, desde os três anos de idade, episódios recorrentes de sibilância com predomínio na primavera e queixas praticamente constantes de obstrução e prurido nasal, sem aparente variação sazonal, condicionando roncopatia noturna nos períodos de agravamento clínico. Apresentou episódio de anafilaxia caracterizado por exantema urticariforme, angioedema e vômitos com a primeira ingestão de papa láctea quando lactente e, posteriormente, com a ingestão de kiwi e episódio de angioedema na primeira e única ingestão de abacate. Encontra-se medicado, desde os 6 anos de idade, diariamente com fluticasona inalada $125 \mathrm{mcg}$, montelucaste $5 \mathrm{mg}$, mometasona intranasal $50 \mu \mathrm{g}$ e, em SOS, salbutamol $300 \mu \mathrm{g} / \mathrm{dose}$, desloratadina $5 \mathrm{mg}$ e adrenalina $0,3 \mathrm{mg} / 0,3 \mathrm{~mL}$. Encontra-se, atualmente, em razoável controle clínico. O exame físico mostra um crescimento estaturoponderal harmonioso, encontrando-se dentro dos percentis 25 e 50 e desenvolvimento psicomotor e nível cognitivo adequados. Dos antecedentes familiares, salienta-se asma alérgica materna, com sensibilização aos ácaros, e rinoconjuntivite alérgica paterna, polissensibilizada.

Da investigação alergológica de rotina, destaca-se:

1. IgE total $1731,0 \mathrm{U} / \mathrm{mL}$;

2. Doseamentos de IgE específica para aeroalergênios positiva para Dermatophagoides pteronysinus (1,33 kU/L), Dermatophagoides farinae $(2,20 \mathrm{kU} / \mathrm{L})$, gramíneas $(>100,0 \mathrm{kU} / \mathrm{L})$, ervas $(52,20 \mathrm{kU} / \mathrm{L})$, árvores $(46,20 \mathrm{kU} / \mathrm{L})$, fungos $(11,50 \mathrm{kU} / \mathrm{L})$, epitélio de gato $(0,76 \mathrm{kU} / \mathrm{L}) \mathrm{e}$ epitélio de cão $(1,90 \mathrm{kU} / \mathrm{L})$; IgE específica para 
alimentos positiva a amendoim $(>100,0 \mathrm{kU} / \mathrm{L})$, clara de ovo $(0,97 \mathrm{kU} / \mathrm{L})$ e proteínas de leite de vaca - caseína $(0,35 \mathrm{kU} / \mathrm{L}), \alpha$-lactoalbumina $(0,45 \mathrm{kU} / \mathrm{L})$ e $\beta$-lactoglobulina $(0,50 \mathrm{kU} / \mathrm{L})$.

3 . Testes cutâneos em picada com extratos comerciais estandardizados (Bial-Aristegui ${ }^{\circledR}$ ) positivos para histamina (4 mm), Dermatophagoides pteronysinus $(6 \mathrm{~mm})$, Dermatophagoides farinae $(6 \mathrm{~mm})$, gramíneas $(9 \mathrm{~mm})$, Alternaria alternata $(4 \mathrm{~mm})$, epitélio de gato $(6 \mathrm{~mm})$, epitélio de cão $(6 \mathrm{~mm})$, Kiwi (6 mm), clara de ovo $(4 \mathrm{~mm})$, frutos secos $(10 \mathrm{~mm})$.

Realizou, igualmente, estudo funcional respiratório com parâmetros dentro da normalidade e índice de Tiffenau de 0,9. Dada complexidade, optou-se pelo estudo alergológico molecular ImmunoCAP ${ }^{\circledR} \mathrm{Isac}$ (Immuno Solid Phase Allergen Chip), descritos nas Tabelas 1 e 2. O ImmunoCAP ${ }^{\circledR}$ Isac é um teste in vitro que procura identificar e determinar a presença de IgE no soro ou no plasma humano para 112 diferentes componentes específicos de espécie e de reatividade cruzada proveniente de 51 fontes alergênicas.

Tabela 1. Resultados de Componentes Específicos de Espécie ImmunoCAP ${ }^{\circledR}$ Isac (Immuno Solid Phase Allergen Chip).

\begin{tabular}{|c|c|c|}
\hline & Proteína & Resultado (ISU-E) \\
\hline \multicolumn{3}{|l|}{ Alimento } \\
\hline Clara de Ovo & nGal d 2 & 0,6 \\
\hline Cajú & rAna o 2 & 0,2 \\
\hline Noz & nJug r 2 & 3,5 \\
\hline \multirow[t]{4}{*}{ Amendoim } & rAra h 1 & 23,0 \\
\hline & rAra h2 & 53,0 \\
\hline & rAra h3 & 8,0 \\
\hline & r Arah 6 & 80,0 \\
\hline \multirow[t]{2}{*}{ Soja } & nGly m5 & 2,7 \\
\hline & nGly m6 & 2,3 \\
\hline \multicolumn{3}{|c|}{ Pólens de gramíneas } \\
\hline Grama & nCyn d1 & 9,1 \\
\hline \multirow[t]{6}{*}{ Rabo de gato } & rPhl p1 & 81,0 \\
\hline & $\mathrm{rPhl}$ p2 & 51,0 \\
\hline & $\mathrm{rPhl}$ p4 & 6,4 \\
\hline & $\mathrm{rPhl}$ p5 & 46,0 \\
\hline & rPhl p6 & 74,0 \\
\hline & rPhl p11 & 18,0 \\
\hline \multicolumn{3}{|l|}{ Pólens de árvores } \\
\hline Vidoeiro & rBet v1 & 13,0 \\
\hline Cedro japonês & nCVry j1 & 3,2 \\
\hline Cipreste & nCup a1 & 2,9 \\
\hline \multirow[t]{2}{*}{ Plátano } & rPla a1 & 0,5 \\
\hline & nPla a2 & 2,7 \\
\hline \multicolumn{3}{|l|}{ Animais } \\
\hline Gato & rFerl d1 & 8,6 \\
\hline \multicolumn{3}{|l|}{ Fungos } \\
\hline Alternaria & rAlt a1 & 16,0 \\
\hline
\end{tabular}

ISU-E, ISAC unidades estandardizadas. ISU-E <0,1: indetectável; ISU-E baixo = 0,1-0,9; ISU-E moderado a alto $=1,0-14,9$; ISU-E muito alto $\geq 15$.
Tabela 2. Resultados de Componentes de Reatividade Cruzada ImmunoCAP ${ }^{\circledR}$ Isac (Immuno Solid Phase Allergen Chip).

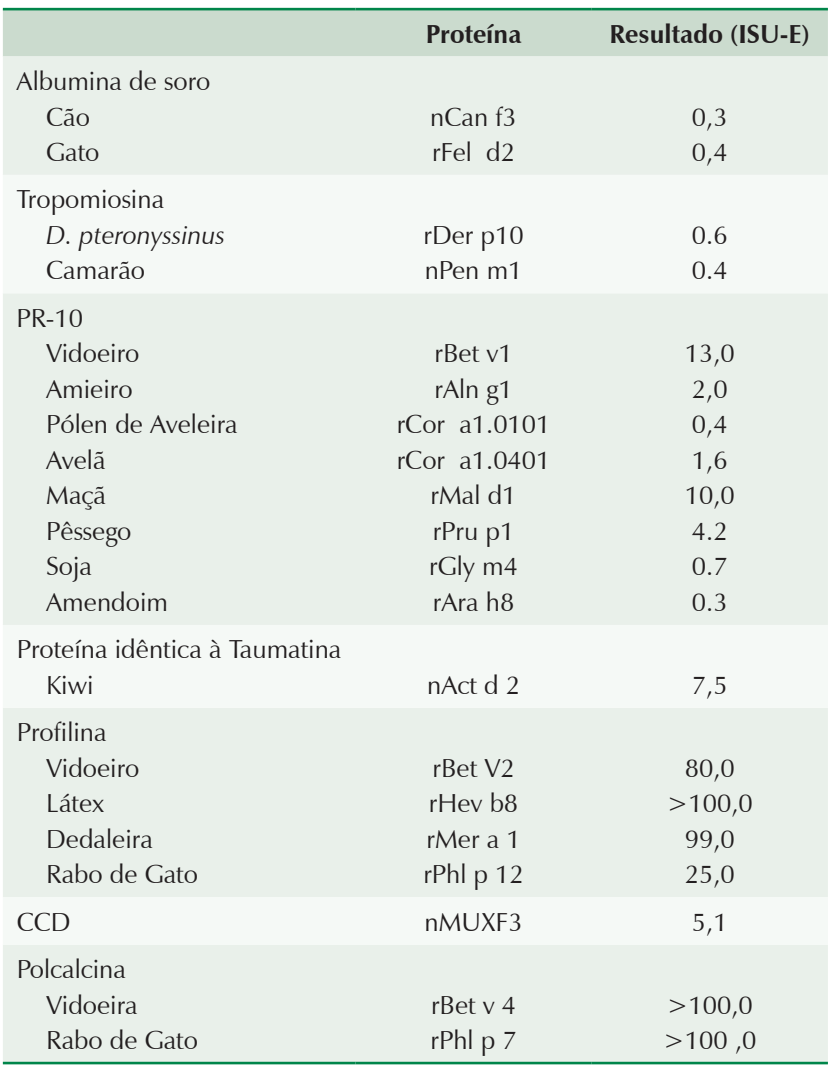

ISU-E, ISAC unidades estandardizadas. ISU-E <0,1: indetectável; ISU-E baixo = 0,1-0,9 ISU-E moderado a alto $=1,0-14,9$; ISU-E muito alto $\geq 15$.

\section{DISCUSSÃO}

Os autores apresentam este caso pela sua complexidade, face ao contexto de sensibilização múltipla. Tratando-se de um doente polissensibilizado complexo, até com antecedentes pessoais de reações alérgicas graves e controle clínico razoável, na atualidade é justificável a realização do estudo alergológico molecular no sentido de melhor percepção do perfil de sensibilização, o que concorre para uma melhor adequação terapêutica.

Do resultado obtido pelo ImmunoCAP ${ }^{\circledR}$ Isac, mantém-se a perceção de que estamos perante um doente complexo, uma vez que foi identificada positividade a 42 alergênios distintos, com uma percentagem significativa de resultados moderadoelevado ou mesmo muito elevado, o que é verdade sobretudo para alergênio específicos dos frutos secos e gramíneas.

No caso particular dos aeroalergénios, salienta-se a elevadíssima positividade a super-famílias proteicas 
como as profilinas e as polcalcinas, grupos alergénicos major presentes nas gramíneas [6], o que condiz com o padrão sazonal de sintomas apresentado pelo doente em estudo e, torna razoável a iniciação de imunoterapia específica para este grupo de alergénios.

As reações descritas com frutos, como o kiwi e o abacate, podem ser justificadas pela presença de proteínas de reatividade cruzada com proteínas presentes nos pólens. As proteínas do grupo das $P R-10$ e Act $d 2$ (proteína idêntica a taumatina) existem também no Kiwi. No caso do abacate, são descritos na literatura dois alergênios presentes ao grupo das profilinas - Pers a 1 (chitinase) e Pers a 4 (profilina). A presença destas proteínas em muitos alimentos de origem vegetal pode justificar reações alérgicas com frutas, nozes e vegetais, por via de reatividade cruzada, estando na maior parte dos casos, associada a sintomas locais e a síndrome oral-alérgico. A maioria destas proteínas é termolábil, indicando que alimentos cozinhados possam ser tolerados.

Também a positividade ao látex advém de reação cruzada com profilinas. A associação de alergia ao látex e alergia alimentar a frutos e outros vegetais com reatividade cruzada com látex, é denominada síndrome látex-frutos, o qual, segundo a literatura mais recente, está presente em 30 a $50 \%$ dos doentes com alergia ao látex [6]. Neste caso em concreto, a positividade ao látex tem apenas implicado o alergénio $\operatorname{Hev} b 8$, um dos mais frequentemente descritos na literatura como responsável por este tipo de síndrome; no entanto, uma vez que o referido alergénio é o único positivo no doente em estudo, e dado não ser um determinante específico de espécie, é com alguma segurança que se pode concluir que este doente não é verdadeiramente alérgico ao látex e que o contacto com este produto não será alvo de aparecimento de sintomatologia alérgica.

Com relação aos frutos secos, encontramos positividade em proteínas específicas de espécie apenas no amendoim (positividade mais significativa) e noz, sendo lícita a liberalização do consumo de outros frutos secos; todos os alergénios encontrados são termoestáveis e, no caso particular do amendoim, Ara h2 e Ara h6 são proteínas que se associam com reações sistémicas e com maior potencial de gravidade, o que também deve ser tido em conta na abordagem do doente em estudo, com medidas de evicção extremamente restritas e prescrição e terapêutica de emergência como a adrenalina subcutânea [6].

A positividade dos testes cutâneos ao marisco deriva da presença de IgE especifica para uma proteína de reação cruzada, a tropomiosina, a qual também condiciona a sensibilização a ácaros do pó doméstico; recomenda-se a evicção deste grupo alimentar da dieta independentemente da forma como é cozinhado, uma vez que também se trata de um grupo proteico termoestável. A sensibilização à tropomiosina está descrita como responsável por reações sistémicas graves mesmo com a ingestão de pequenas quantidades de marisco e, inclusive pode, por reação cruzada, manifestar-se perante outras espécies de crustáceos e moluscos [6].

Em relação às proteínas do leite de vaca, dada negatividade dos resultados do estudo molecular por contraponto aos resultados dos testes de primeira linha, justifica-se a realização de uma prova de provocação oral em meio hospitalar [6].

Apesar do estudo imunoalergológico exaustivo, que permitiu identificar e objetivar a sensibilização múltipla a alergênios alimentares e aeroalergênios, e instituir medidas de controlo direccionadas, a multiplicidade de fontes alergênicas não permite assegurar a completa estabilidade clínica do doente. A ingestão ou contacto acidental recorrente com os diferentes alergénios a que está sensibilizado pode assumir gravidade preocupante.

Assistiu-se nas últimas décadas a uma revolução na vertente diagnóstica em imunoalergologia uma vez que, em virtude dos avanços científicos ao nível da biologia molecular, atualmente o estudo por $\operatorname{IgE}$ específicas poderá ser dirigido a componentes proteicos (naturais ou recombinantes) dos principais alergénios existentes. No caso descrito, o estudo molecular foi fundamental para definir o perfil alergológico do doente.

\section{NOTAS}

Apoio financeiro

Este estudo não recebeu apoio financeiro de fontes externas.

Declaração de conflito de interesses

Os autores declaram não haver conflitos de interesses relevantes ao conteúdo deste estudo.

Contribuições dos autores

Todos os autores fizeram contribuições substanciais para concepção, ou delineamento, ou aquisição, ou análise ou interpretação de dados; e redação do trabalho ou revisão crítica; e aprovação final da versão para publicação.

Disponibilidade dos dados e responsabilidade pelos resultados

Todos os autores declaram ter tido total acesso aos dados obtidos e assumem completa responsabilidade pela integridade destes resultados. 


\section{REFERÊNCIAS}

1. van Hage M, Hamsten C, Valenta R. ImmunoCAP assays: pros and cons in allergology. J Allergy Clin Immunol. 2017;140(4):974-7. https://doi.org/10.1016/j.jaci.2017.05.008

2. Alessandri C, Zennaro D, Zaffiro A, Mari A. Molecular allergology approach to allergic diseases in the paediatric age. Ital J Pediatr. 2009;35(1):29. https://doi.org/10.1186/1824-7288-35-29

3. Melioli G, Savi E, Crivellaro MA, Passalacqua G. Potential of molecular based diagnostics and its impact on allergen immunotherapy. Asthma Res Pract. 2016;2:9. https://doi.org/10.1186/s40733-016-0024-8

4. Hamilton RG, Kleine-Tebbe J. Molecular allergy diagnostics: analytical features that support clinical decisions. Curr Allergy Asthma Rep. 2015;15(9):57. https://doi.org/10.1007/s11882-015-0556-7

5. Arruda LK, Moreno AS, Ferreira F. Diagnóstico molecular de alergia: pronto para a prática clínica? Braz J Allergy Immunol. 2013;1(4):187-94. https://doi.org/10.5935/2318-5015.20130024

6. Matricardi PM, Kleine-Tebbe J, Hoffmann HJ, Valenta R, Hilger C, Hofmaier S, Aalberse RC, Agache I, Asero R, Ballmer-Weber B, Barber D, Beyer K, Biedermann T, Bilò MB, Blank S, Bohle B, Bosshard PP, Breiteneder H, Brough HA, Caraballo L, Caubet JC, Crameri R, Davies JM, Douladiris N, Ebisawa M, EIgenmann PA, FernandezRivas M, Ferreira F, Gadermaier G, Glatz M, Hamilton RG, Hawranek T, Hellings P, Hoffmann-Sommergruber K, Jakob T, Jappe U, Jutel M, Kamath SD, Knol EF, Korosec P, Kuehn A, Lack G, Lopata AL, Mäkelä M, Morisset M, Niederberger V, Nowak-Węgrzyn AH, Papadopoulos NG, Pastorello EA, Pauli G, Platts-Mills T, Posa D, Poulsen LK, Raulf M, Sastre J, Scala E, Schmid JM, Schmid-Grendelmeier P, van Hage M, van Ree R, Vieths S, Weber R, Wickman M, Muraro A, Ollert M. EAACi molecular allergology user's guide. Pediatr Allergy Immunol. 2016;27 Suppl 23:1-250. https://doi.org/10.1111/pai.12563 